\title{
Modulated AlHT Image Contrast Enhancement Algorithm based on Contrast-Limited Adaptive Histogram Equalization
}

\author{
Cheng-Yi Yu ${ }^{1}$, Hsueh-Yi Lin ${ }^{1, *}$,Yen-Chieh Ouyang ${ }^{2}$ and Tzu-Wei Yu \\ ${ }^{1}$ Dept. of Computer Science and Information Engineering, Chin-Yi University of Technology, Taichung, Taiwan, ROC \\ ${ }^{2}$ Dept. of Electrical Engineering, National Chun- Hsing University, Taichung, ROC \\ ${ }^{3}$ Dept. of Electronic Engineering, Chin-Yi University of Technology, Taichung, Taiwan, ROC
}

Received: 2o Oct. 2012, Revised: 2 Feb. 2013, Accepted: 4 Feb. 2013

Published online: 1 Jun. 2013

\begin{abstract}
The Adaptive Inverse Hyperbolic Tangent (AIHT) algorithm is capable of enhancing the global contrast of the original image adaptively, while extruding the details of objects simultaneously, cannot enhance local contrast. Therefore, we proposed a ContrastLimited Adaptive Histogram Equalization (CLAHE) modulation based on AIHT contrast enhancement algorithm from conjugate AIHT and CLAHE image contrast enhance algorithm. The CLAHE has good contrast enhance performance, but excessive contrast enhance will produce the serious chromatic aberration results. We apply the AIHT and CLAHE advantage to present a joint multiple processes algorithm of contrast enhancement to achieve better contrast enhancement effect.
\end{abstract}

Keywords: Contrast Enhancement, Global Contrast Enhancement, Local Contrast Enhancement, Adaptive Inverse Hyperbolic Tangent (AIHT), Contrast-Limited Adaptive Histogram Equalization (CLAHE)

\section{Introduction}

The human visual system can distinguish only about 20 to 30 shades of gray under a given adaptation level. Under the same conditions it discriminates a much larger number of color hues. Because of the human visual perception is insensitive to shades of gray. Therefore, contrast enhancement addresses the problem of improving the contrast in an image in order to make various features more easily perceived. Generalizations of this method use multiple histograms to emphasize local contrast, rather than overall contrast. Examples of such methods include adaptive histogram equalization and contrast limiting adaptive histogram equalization or CLAHE.

One of the challenges of image Luminance is acquiring sufficient signal for good color data. This is especially true for dim objects in which the color data has a very low signal/noise ratio with reasonable exposure times. In these cases the unprocessed luminance will often tend to overwhelm and washout the RGB data resulting in muted colors. Attempts to improve the color by increasing saturation or by lowering the opacity of the luminance can result in excessive noise and/or sacrifice of detail and an unsatisfying aesthetic result.

Color distortion, sometimes produced by the contrast enhancement, is a serious problem. In image enhancement, a good algorithm choice can produce images where each object has a determined spectral range. Color distortion starts to be a problem when objects with different spectral characteristics have similar colors after image enhancement. In order to improve above chromatic aberration problem, we presented CLAHE modulation based on MSAIHT contrast enhancement (MSAIHT $\oplus$ CLAHE) algorithm. We apply the MSAIHT and CLAHE advantage to present a joint multiple processes algorithm of contrast enhancement to achieve better contrast enhancement effect. We proposed a CLAHE modulation based on AIHT contrast enhancement algorithm from conjugate AIHT and CLAHE image contrast enhance algorithm.

The remainder of this paper is organized as follows: Section 2 reviews previous work done in the literary. Section 3 develops the CLAHE modulation based on

* Corresponding author e-mail: hyl@ncut.edu.tw 
AIHT contrast enhancement algorithm. Section 4 conducts experiments including simulations. Finally, Section 5 provides future directions of further research.

\section{Contrast Enhancement for an Image}

\subsection{Adaptive Inverse Hyperbolic Tangent Algorithm}

The AIHT enhancement algorithm is an adaptive adjustment of the Inverse Hyperbolic Tangent (IHT) function determined by each pixel's radiance. The bias(x) and gain(x) parameters control the shape of the IHT function $[1,2]$. The AIHT algorithm uses the bias(x) to the power of $\mathrm{x}$ to speed up changing. The gain(x) function is a weighting function which is used to determine the steepness of the AIHT curve. It has several desirable properties. For very small and very large luminance values, its logarithmic function enhances the contrast in both dark and bright areas of an image. Because this function is an asymptote, the outputs are always bounded between 0 and 1[3]. Another advantage of using this function is that it supports an approximately inverse hyperbolic tangent mapping for intermediate luminance, or luminance distributed between dark and bright values $[4,5,6]$. The contrast of an image can be enhanced using adaptive inverse hyperbolic function via the following function:

$$
\operatorname{Enhance}\left(x_{i j}\right)=\left(\log \left(\frac{1+x_{i j}^{\operatorname{bias}(x)}}{1-x_{i j}^{\operatorname{bias}(x)}}\right)-1\right) \times \operatorname{gain}(x)
$$

where $\mathrm{x}_{i j}$ is the image gray level of the $\mathrm{i}^{\text {th }}$ row and $\mathrm{j}^{\text {th }}$ column.

The value of bias controls the centering of the inverse hyperbolic tangent and it's seamlessly scale. It is defined by

$$
\operatorname{bias}(x)=\left(\frac{\operatorname{mean}(x)}{0.5}\right)^{0.25}=\left(\frac{\frac{1}{m \times n} \sum_{i=1}^{m} \sum_{j=1}^{n} x_{i j}}{0.5}\right)^{0.25}
$$

The gain function determines the steepness of the AIHT curve. A gain function is defined by

$$
\operatorname{gain}(x)=0.1 \times(\operatorname{variance}(x))^{0.5}=0.1 \times\left(\frac{1}{m \times n} \sum_{i=1}^{m} \sum_{j=1}^{n}\left(x_{i j}-\mu\right)\right)^{0.5}
$$

Where

$$
\mu=\frac{1}{m \times n} \sum_{i=1}^{m} \sum_{j=1}^{n} x_{i j}
$$

The AIHT-based image contrast enhancement method has two drawbacks. One is that it lacks of a mechanism to adjust the degree of enhancement. Using the AIHT-based image contrast enhancement methods can not retain the subtle brightness distribution of the original image; therefore it may lead to a distortion. Another is that using the AIHT-based algorithm can only be used for the global contrast enhancement and cannot achieve for a local contrast enhanced. Therefore, it is unable to meet the Human Visual System mapping curve and to produce a non-smooth or distorted images phenomenon.

\subsection{Adaptive Histogram Equalization (AHE)}

Adaptive histogram equalization involves selecting a local neighborhood centered around each pixel, calculating and equalizing the histogram of the neighborhood, and then mapping the centered pixel based on the new equalized local histogram $[7,8]$. For example, at each point in an input image we could consider an $8 \times 8$ window around that point. The 64-element histogram could then be used to determine a mapping function to histogram equalize that point based on the neighborhood. Since each point would be based on its own neighborhood, the mapping function can vary over the image. The enhancement is thereby reduced in very uniform areas of the image, which prevents over enhancement of noise and reduces the edge-shadowing effect of unlimited AHE.

\subsection{Contrast-Limited Adaptive Histogram Equalization (CLAHE)}

CLAHE is a special case of the histogram equalization technique [9] that functions adaptively on the image to be enhanced. CLAHE is a technique used to improve the local contrast of an image. The CLAHE is an improved version of AHE, both of which overcome the limitations of standard histogram equalization. It is a generalization of adaptive histogram equalization and ordinary histogram equalization [10], where the histogram is calculated for the contextual region of a pixel. The pixel's intensity is thus transformed to a value within the display range proportional to the pixel intensity's rank in the local intensity histogram. The CLAHE was originally developed for medical imaging and has proven to be successful for enhancement of low-contrast images such as portal films $[11,12,13,14,15]$.

The two primary features is AHE, which divides the images into regions and performs local $\mathrm{HE}$, and the CLAHE, which reduces noise by partially reducing the local HE. Bilinear interpolation is used to avoid visibility of region boundaries. The major problem with CLAHE methods is that it often over enhances the image by creating so called contrast objects that were not visible in the original image. The enhanced image often does not 
look natural and is disturbing [16]. Figure 2.1 show processed image by CLAHE algorithm, chromatic aberration can be seen clearly.

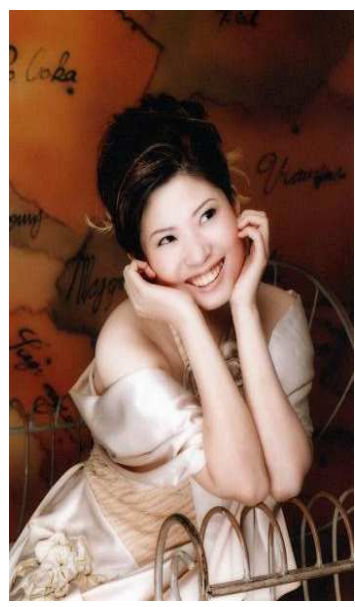

(a)

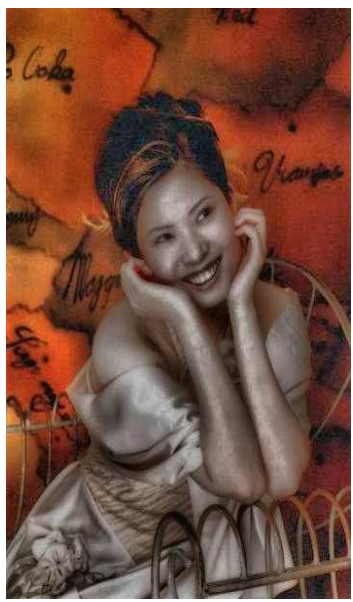

(b)
Fig. 2.1: The CLAHE has chromatic aberration problems. (a) Original image. (b) Processed result by CLAHE algorithm.

\section{CLAHE Modulation Based on AIHT Contrast Enhancement Algorithm}

\subsection{Image fusion}

A general definition of image fusion is given as "Image fusion is the combination of two or more different images to form a new image by using a certain algorithm" $[17,18$, 19]. It aims at obtaining information of greater quality; the exact definition of 'greater quality' will depend upon the application [20]. The linear fusion approach is to simply combine two images into a single image by using a linear equation as equation (3.1):

$$
I_{\text {fused }}(x, y)=\alpha I_{\text {method_A }}(x, y)+(1-\alpha) I_{\text {method_B }}(x, y)
$$

where $\alpha$ is a constant ranging from 0 to 1.0. Since $\alpha$ is less than or equal to 1 , the contrast of the fused image is at most equal to but not greater than the contrasts of the processed image by method $\mathrm{A}$ and the processed image by method B.

\subsection{CLAHE Modulation Based on AIHT Contrast Enhancement (AIHT $\oplus$ CLAHE) Algorithm}

In order to improve observation quality, we presented CLAHE modulation based on AIHT contrast enhancement (AIHT $\oplus$ CLAHE) algorithm. We apply multiple process concepts to image contrast enhancement, and its processed result has a similar modulation effect. Figure 3.1 shows the mapping curve of multiple processes is depend upon AIHT mapping curve. The curve of multiple process is depend upon AIHT mapping curve and more suitable for human perception.

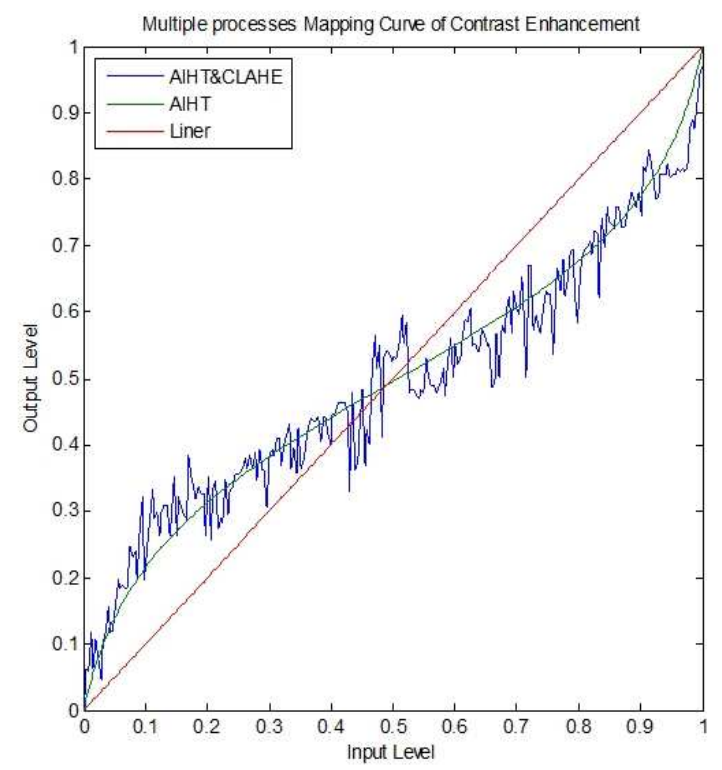

Fig. 3.1: The mapping curve of multiple processes is depend upon AIHT mapping curve.

Figure 3.2 shows a block diagram of CLAHE Modulation based on AIHT image contrast enhancement algorithm. The intensity component is detached from the color information. Figure 3.3 shows a processing procedure of AIHT and CLAHE by Multiple Processes algorithm. The CLAHE modulation based on AIHT contrast enhancement technique consists of two steps multiple process and fusion of weight ratio step. First, we applied multiple processes step to the image to enhance processing. In enhancement step was applied to the AIHT and CLAHE algorithm and bring out hidden details and enhance contrast, respectively.

Second, Then the enhanced result will be multiply by $\alpha$ and $\beta$ of weight parameter, respectively, and integrate output of both multiplied result. The enhanced output image Enhance_Multiple integrate output of both AIHT and CLAHE result by $\alpha$ and $\beta$ of weight parameter, 
respectively. The Enhance Multiple approach for processing input image $\mathrm{x}$, is described by equation (3.2):

$$
\text { Enhance Multiple }=\alpha \times \text { AIHT }(x)+\beta \times C L A H E(x)
$$

where $\alpha$ and $\beta$ is less the weight of AIHT and CLAHE, respectively, $\mathrm{x}$ is luminance of input image. The $\alpha$ and $\beta$ have to satisfied $\alpha+\beta=1$, and that $0 \leq \alpha \leq 1$ and $0 \leq \beta \leq 1$.

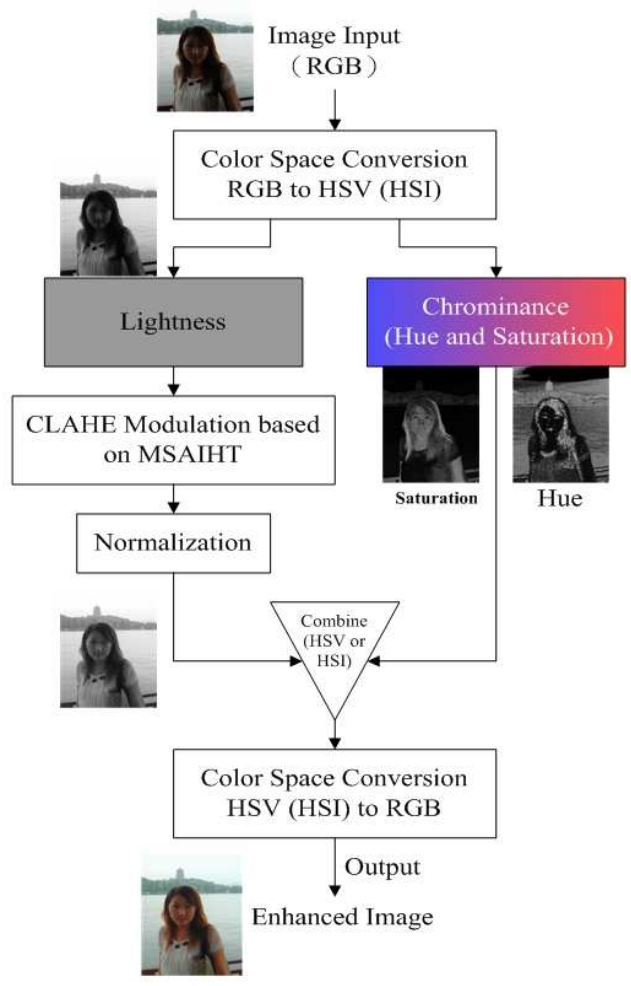

Fig. 3.2: A flowchart of CLAHE modulation based on AIHT image contrast enhancement algorithm.

\section{Implementation and Experimental Results}

Various types of histogram distributions images underexposures, midexposures, and overexposures images- were tested under this proposed method. These images include some poor contrast types of daily life images which can not be fixed by conventional contrast methods. Those images can be further categorized into outdoor and indoor images. The above images belong to four extreme types of images which are dark image, bright image, back-lighted image, and low-contrast image. The conventional contrast enhancement algorithms and the proposed enhancement algorithm have been implemented in Matlab code of MathWorks version

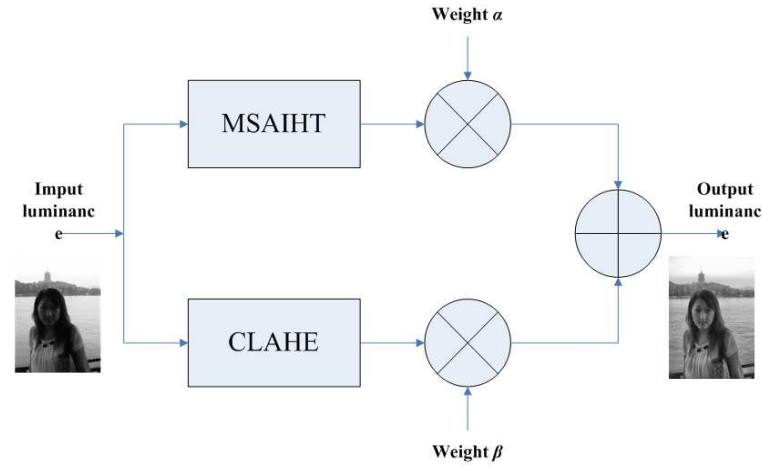

Fig. 3.3: A processing procedures of AIHT and CLAHE by multiple processes algorithm.

R2010a. A $2.83 \mathrm{GHz}$ Intel Core 2 Quad PC with 2GB RAM of hardware platform was used for this simulation. The processed result has similar Amplitude Modulation (AM) effect of signal processing conveys information over a carrier wave by varying its instantaneous frequency. As figure 3.1 show mapping curve of multiple process is depend upon AIHT mapping curve and more suitable for human perception. Figure 4.1 shows the processed result of CLAHE modulation based on AIHT algorithm by fusion of varies weight ratio.

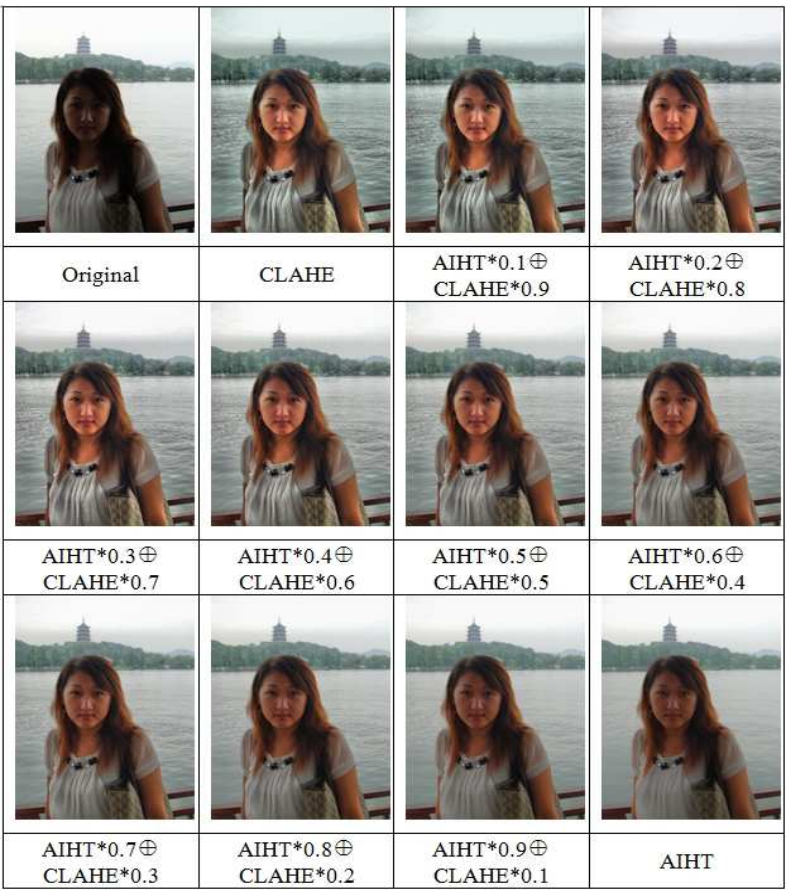

Fig. 4.1: The compare results of the outdoor images processed by AIHT method at different weight ( $\alpha$ and $\beta$ ) parameters. 
Comparing figure 4.1 ongoing processing results, we can choose of best weight parameters with $\alpha=0.5, \beta=0.5$ and $\alpha+\beta=1$. The processed results have a greater global AIHT contrast enhancement when the parameter $\alpha>\beta$, and produced a lower local contrast enhancement. Oppositely, the processed results have a greater local CLAHE contrast enhancement when the parameter $\alpha<\beta$, and produced the serious chromatic aberration. Figure 4-2 shows the results by used the same weight parameters value with $\alpha=0.5$ and $\beta=0.5$. The method mapping curve of AIHT method is suitable for human perception where we can see is a global contrast enhancement mean while the CLAHE method, lie on the AIHT mapping curve, can do the local contrast enhancement job.

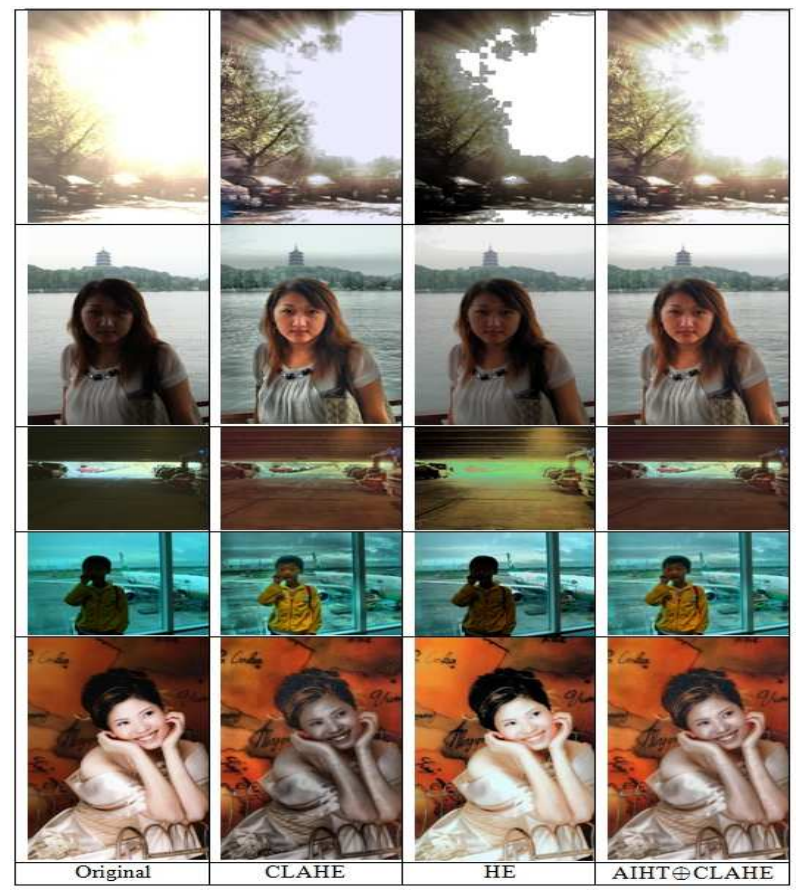

Fig. 4.2: The compare results of the various images processed by AIHT method at different weight ( $\alpha$ and $\beta$ ) parameters.

Experimental results demonstrate of the AIHT $\oplus$ CLAHE method is capable to improve AIHT algorithm alone where they are lacking of the local contrast enhancement capability and also make a correction for CLAHE where it may cause the excessive contrast enhancement and produced a serious chromatic aberration problem. In summary, the proposed AIHT $\oplus$ CLAHE algorithm outperforms conventional (HE and CLAHE) and AIHT algorithms for the contrast and details of image.

\section{Conclusions}

We apply the AIHT and CLAHE advantage to present a CLAHE modulation based on AIHT contrast enhancement algorithm from conjugate AIHT and CLAHE image contrast enhance algorithm. These proposed algorithms based on AIHT algorithm as a contrast function to map from the original image into a transformed image. Use of this algorithm can improve displayed quality of contrast in the scenes and offers an efficiency way for fast computation. Experimental results show AIHT $\oplus$ CLAHE algorithm have more better local contrast enhancement effect and show details than AIHT and CLAHE.

\section{References}

[1] C. Y. Yu, Y. C. Ouyang, C. M. Wang, C. I. Chang, Z. W. Yu, "Contrast Adjustment in Displaying Scenes Using Inverse Hyperbolic Function," 2009 The 22th IPPR Conference on Computer Vision, Graphics, and Image Processing, pp.10201027, 2009.

[2] C. Y. Yu, Y. C. Ouyang, C. M. Wang and C. I. Chang, "Adaptive Inverse Hyperbolic Tangent algorithm for Dynamic Contrast Adjustment in Displaying Scenes," EURASIP Journal on Advances in Signal Processing, June 2010

[3] K. I. Naka and W. A. Rushton, "S-potentials from luminosity units in the retina of fish (cyprinidae)," Journal of Physiology, vol. 185, no. 3, pp. 587-599, 1966.

[4] J. Kleinschmidt and J. E. Dowling, "Intracellular recordings from gecko photoreceptors during light and dark adaptation," Journal of General Physiology, vol. 66, no. 5,pp.617-648, 1975.

[5] D. C. Hood and M. A. Finkelstein, "A comparison of changes in sensitivity and sensation: implications for the responseintensity function of the human photopic system”, Journal of Experimental Psychology?Human Perception and Performance, vol. 5, no. 3, pp. 391-405, 1979.

[6] D. C. Hood, M. A. Finkelstein, and E. Buckingham, "Psychophysical tests of models of the response function," Vision Research, vol. 19, no. 4, pp. 401-406, 1979.

[7] S. M. Pizer, E. P. Amburn, J. D. Austin, R. Cromartie, A. Geselowitz, T. Geer, B. H. Romeny, J. B. Zimmerman, and K. Zuiderveld, "Adaptive histogram equalization and its variations," Computer Vision, Graphics, and Image Processing, 39(3): pp. 355-368, September 1987.

[8] A. P. Stefanoyiannis, L. Costaridou, S. Skiadopoulos, G. Panayiotakis (2003), "A digital equalization technique improving visualization of dense mammary gland and breast periphery in mammography," Eur J Radiol, Vol. 45, pp. 139149.

[9] R. C. Gonzalez, and R. E. Woods, 1992, "Digital Image Processing," Addison-Wesley.

[10] S. Pizer, J. B. Zimmerman, E. V. Staab, "Adaptive grey level assignment in CT scan display," J Comput Assist Tomogr, pp. 300-305, 1984.

[11] Zuiderveld and Karel (1994), "Contrast limited adaptive histogram equalization,” Graphics gems IV, Academic Press Professional, Inc., pp. 474-485. 
[12] A. Pratt, and K. William, "Digital image processing," Wiley, N.Y., 1978.

[13] A. Daskalakis, D. Cavouras, P. Bougioukos, S. Kostopoulos, P. Georgiadis, I. Kalatzis, G. Nikiforidis, "An efficient CLAHE-based, Spot-adaptive, image segmentation technique for improving microarray genes quantification," 2nd International Conference on Experiments /Process/System Modelling/Simulation \& Optimization, July, 2007.

[14] S. Srinivasan, N. Balram, "Adaptive Contrast Enhancement Using Local Region Stretching,” Proc.of ASID’06, 8-12 Oct, New Delhi, pages 152-155.

[15] J. Rosenman, C. A. Roe, R. Crommartie, K. E. Muller, and S. M. Pizer, "Portal film enhancement: Technique and clinical utility," Int. J. Radiat. Oncol. Biol. Physics, pages 333-338, 1993.

[16] E. D. Pisano, S. Zong, B. M. Hemminger, M. DeLuca, R. E. Johnston, K. Muller, M. P. Braeuning, S. M. Pizer (1998), "Contrast limited adaptive histogram equalization image processing to improve the detection of simulated spiculations in dense mammograms," J Digit Imaging, Vol. 11, pp. 193-200.

[17] L. Wald, "Some terms of reference in data fusion," IEEE Transactions on Geoscience and Remote Sensing, vol. 37, pp. 1190-1193, May 1999.

[18] J. J. Clark and A. L. Yuille, "Data fusion for sensory information processing systems," Kluwer Academic Publishers, 1990.

[19] J. L. Genderen and C. Pohl, "Image fusion: Issues, techniques and applications," Strasbourg, France, pp. 18-26, September 1994.

[20] J. A. Stark, "Adaptive image contrast enhancement using generalizations of histogram equalization," IEEE Transactions on Image Processing, vol. 9, no. 5, pp. 889-896, 2000 .

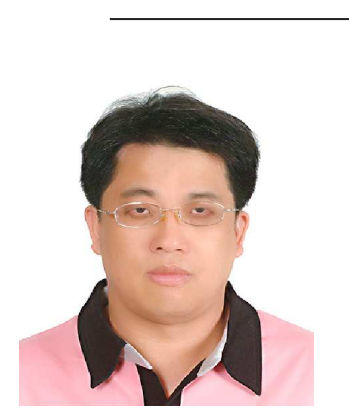

Cheng-Yi Yu was born in May 2, 1969 in Taichung County, Taiwan. He received his Master (2003) and $\mathrm{Ph}$. D (2011) degree in Electrical Engineering from the National Chung Hsing University, Taichung, Taiwan, R.O.C. Currently, he is a Assistant Professor of Department of Computer Science and Information Engineering of National Chin-Yi University of Technology. His research interests include image contrast enhancement, image processing and digital signal processing.
Hsueh-Yi

Lin

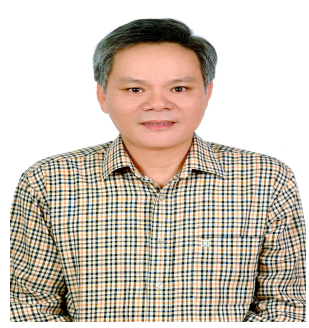

was born in May 10, 1959 in I-Lan County, Taiwan. He obtained Master (1991) degree in Computer Science from the Northrop University, California USA. Currently, he is a Assistant Professor of Department of Computer Science and Information Engineering of National Chin-Yi University of Technology. His research interests in image processing and computer network.

\section{Yen-Chieh}

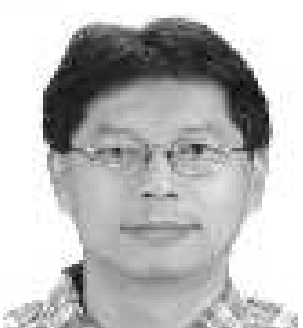

Ouyang Yen-Chieh Ouyang (S'86-M'92) received the B.S.E.E. degree in electrical engineering from Feng Chia University, Taiwan, Taichung, R.O.C., in 1981, and the M.S. and Ph.D. degrees in electrical engineering from the University of Memphis, Memphis, TN, in 1987 and 1992, respectively. Since 1992, he has been with the Department of Electrical Engineering, National Chung Hsing University, Taichung, where he is currently a Professor and the Director of the Multimedia Communication Laboratory. His current research interests include hyperspectral image processing, medical imaging, communication networks, network security in mobile networks, multimedia system design, and performance evaluation.

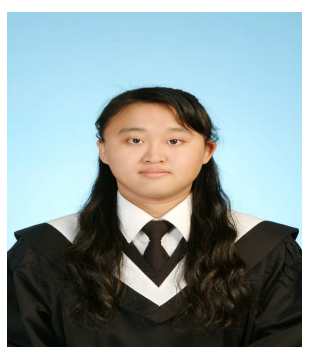

Tzu-Wei Yu was born in Taichung, Taiwan. She received the M.S. degree in Department of Electronic Engineering

Chin-Yi University of Technology Taichung, Taiwan R.O.C. Her areas of interest are image contrast enhancement, image processing. 